\title{
UNIDADE RETIFICADORA TRIFÁSICA ISOLADA COM ALTO FATOR DE POTÊNCIA
}

\author{
Denizar Cruz Martins e Elias Sebastião de Andrade \\ Universidade Federal de Santa Catarina \\ Departamento de Engenharia Elétrica \\ INEP - Instituto de Eletrônica de Potência \\ Caixa Postal 5119 \\ 88040-970 - Florianópolis, SC - Fone: (48)331.9204 - E-mail: denizar@inep.ufsc.br
}

\begin{abstract}
Resumo - Este artigo apresenta a análise de um conversor trifásico isolado operando em comutação suave para aplicação em carregamento de baterias. A estrutura trabalha com alto fator de potência sem a necessidade de circuitos intermediários. Suas principais características são: simplicidade no circuito de comando e controle das chaves estáticas controladas, robustez do circuito de potência e alta confiabilidade. Princípio de operação, análise matemática, procedimento de projeto e resultados experimentais obtidos a partir de um protótipo de laboratório (48V/55A) são apresentados.
\end{abstract}

Abstract - This paper presents the analysis of an isolated three-phase converter operating in soft commutation as a battery charger. The structure works with a high power factor without intermediate circuits. Its main features are: simplicity of the control and drive circuit, and robustness of the power circuit. Principles of operation, simulation and experimental results obtained from a laboratory prototype $(48 \mathrm{~V} / 55 \mathrm{~A})$ are presented.

\section{INTRODUÇÃO}

Nos últimos vinte anos a Eletrônica de Potência tem alcançado um grau de desenvolvimento técnico e científico surpreendente, tanto no que se refere à criação de variações topológicas como no aperfeiçoamento das estratégias de comando e controle dos conversores estáticos.

Devido a esse enorme desempenho, os conversores estáticos são normalmente utilizados nas mais variadas aplicações industriais, e se constituem atualmente em um dos maiores temas de estudo em engenharia elétrica.

Apesar desse extraordinário desempenho, o estágio de entrada dos conversores estáticos nos quais se emprega um retificador a diodo em ponte acoplado a um filtro capacitivo com capacitância de valor expressivo, apresenta alguns sérios problemas para a rede elétrica de alimentação. A associação desses componentes gera uma carga não linear que, quando conectada ao sistema elétrico de potência causa sérias distorções na corrente de entrada, resultando em um elevado conteúdo harmônico.

Devido a esse inconveniente, as pesquisas na área de conversores CA-CC trifásicos com alto fator de potência têm sido intensificadas, como mostram as referências [1-7]. Para o sucesso da pesquisa, as estruturas propostas devem apresentar algumas peculiaridades tais como: conversão da tensão alternada de entrada em tensão contínua regulada e isolada na saída, comutação suave, e o menor número possível de interruptores controlados.

Em [1] tem-se como vantagem a utilização de conversores independentes, facilitando o projeto e aumentando a confiabilidade do sistema. Contudo, a transferência de energia é realizada em dois estágios, onde o primeiro estágio consiste em um pré-regulador de fator de potência. Além disso, o fluxo de potência é pulsado e a estrutura apresenta uma elevada quantidade de componentes.

Os trabalhos apresentados em [2 - 4] mostram um fluxo de potência constante, entretanto, os circuitos de comando e controle dos interruptores estáticos são complexos e a transferência de energia é ainda realizada em dois estágios. Os conversores não são independentes, comprometendo a confiabilidade do sistema. Em [5 - 7] melhores resultados são obtidos em termos de rendimento do circuito, operando com fluxo de potência constante; porém, ainda apresentam grandes dificuldades ao nível de implementação dos circuitos de comando, devido à sua complexidade, e a transferência de energia se dá também em dois estágios.

A estrutura de potência do conversor proposto neste trabalho é particularmente simples e permite o projeto de um equipamento robusto com baixo custo, devido às seguintes razões: não há a necessidade de filtros de baixa freqüência na entrada e tampouco na saída; as perdas durante a comutação são praticamente nulas; o circuito de comando é muito simples; cada fase representa um conversor que opera independentemente dos outros, com um único estágio de processamento de energia; e o sistema não necessita de préregulador para correção do fator de potência.

Com essa topologia obtém-se as características fundamentais para a aplicação desejada, ou seja, isolamento galvânico entre a fonte de entrada e o conversor, simplicidade do circuito de comando, possibilidade do controle do fluxo de potência através da modulação da freqüência agindo sobre dois interruptores por fase, característica de saída como fonte de corrente, e baixa ondulação de corrente na saída. Há ainda uma outra importante característica desse conversor, qual seja, o alto fator de potência de entrada sem a necessidade de circuitos intermediários de correção.

\section{CIRCUITO PROPOSTO}

A literatura tem apresentado soluções para a correção do fator de potência em retificadores trifásicos, baseados em conversores estáticos, cujas estratégias podem ser sumarizadas conforme descrito abaixo: 
a) Três conversores monofásicos com dois estágios de processamento de energia [1]: Nesta estratégia os conversores de cada fase são independentes permitindo o uso de módulos padronizados. No caso de falha de qualquer módulo o sistema continua operando com menor potência. Contudo, a utilização de dois estágios acarreta uma quantidade excessiva de componentes;

b) Um conversor trifásico com dois estágios de processamento de energia [2 - 7]: Nesta estratégia o número de componentes é menor, embora se utilize dois estágios de potência, por outro lado a utilização de um único conversor trifásico compromete a confiabilidade do sistema.

A proposta apresentada neste artigo consiste na utilização de conversores independentes para cada fase empregando um único estágio de processamento de energia. Pretende-se, dessa forma, preservar a confiabilidade do sistema com um número reduzido de componentes. Além disso, particular atenção foi dada para que o circuito de comando seja simples e de fácil implementação.

\section{A. Descrição do Circuito Proposto}

O conversor possui na entrada um retificador em ponte completa seguido por um circuito série ressonante com tensão grampeada pelo capacitor ressonante. O diagrama de potência do conversor ressonante proposto está representado na figura 1 onde:
vac tensão alternada de entrada;
Eo
D1-D12 tensão nas baterias;
diodos retificadores de saida;
DG1-DG6 diodos grampeadores de tensão;
T1-T6 interruptores estáticos controlados;

Lr1-Lr3 indutores ressonantes;

$\mathrm{Cr} 1-\mathrm{Cr} 3$ capacitores ressonantes.

Os interruptores T1, T3 e T5 são comandados simultaneamente, assim como T2, T4 e T6. Por esse motivo o circuito de comando se torna muito simples. Na figura 5, a duração do sinal de comando para T1 e T2 é mostrada durante um período de chaveamento.

\section{B. Princípio de Operação}

A operação deste conversor é baseada em alguns princípios básicos, tais como: 1) a freqüência de chaveamento é bem superior à freqüência da rede de entrada; 2) a tensão da bateria $E o$ é muito menor do que a tensão de pico da fonte de entrada $V m$, assim $\theta_{l}$ (ângulo inicial) é muito pequeno; e 3) o estágio inversor opera em regime permanente com freqüência constante.

Para um quarto do período da tensão de entrada, há dois diferentes modos de operação, como está mostrado na figura 2.

Modo $A$ : $\left(0<\theta<\theta_{l}\right) v a c<2$.Eop. Não há transferência de energia para a carga.

Modo B: $\left(\theta_{l}<\theta<\pi / 2\right)$. Durante este intervalo, o conversor transfere energia à carga.

Portanto:

$$
\begin{aligned}
& \sin \theta_{1}=\left(\frac{2 \cdot E o p}{V m}\right) \Rightarrow \theta_{1}=\arcsin (q) \\
& \cos \theta_{1}=\sqrt{1-\sin ^{2} \theta_{1}}=\sqrt{1-q^{2}}
\end{aligned}
$$

O parâmetro $q$ é definido como o ganho estático de tensão dado pela relação $(2 . E o p / V m)$, e Eop representa a tensão no secundário do transformador refletida ao primário.

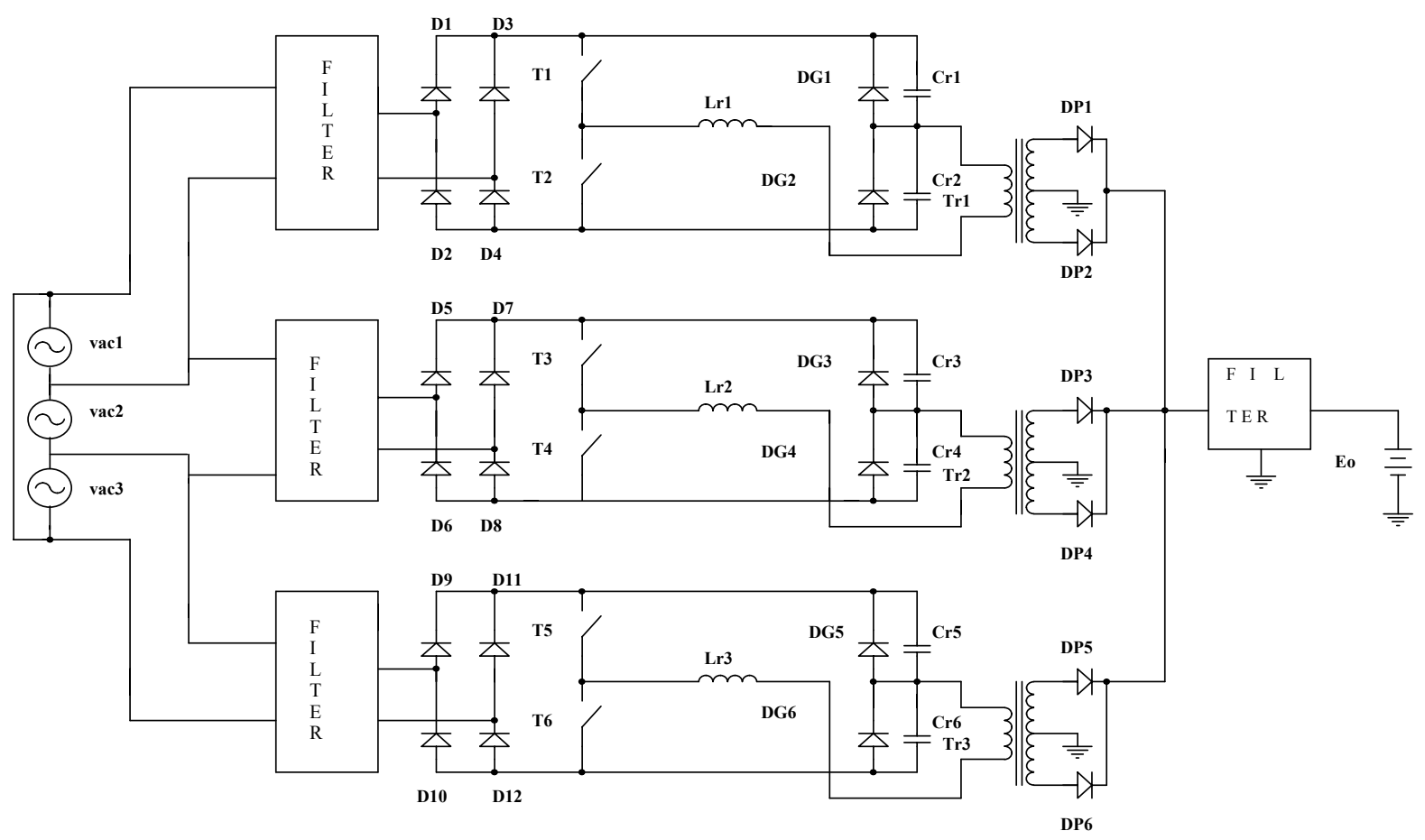

Figura 1: Circuito de potência proposto. 


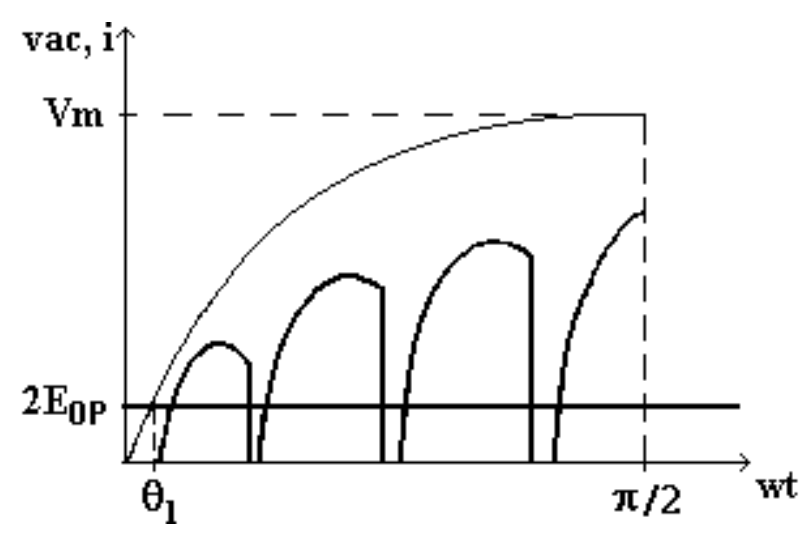

Figura 2: Tensão e corrente de entrada.

\section{Etapas de Operação}

Durante um período de chaveamento do conversor ressonante, a tensão de entrada $v_{a c}$ pode ser considerada constante. Portanto, a estrutura a ser analisada é mostrada na figura 4, onde as tensões Vin e Eop são consideradas constantes e todos os componentes são tomados como ideais. As etapas de operação apresentadas a seguir estão também representadas na figura 4.

$1^{\underline{a}}$ Etapa (to, $t 1 \rightarrow$ ressonante): T1, D3, D6 estão conduzindo:

Condições iniciais: $i_{L r}=0 ; \quad v_{C r 1}=V i n ; \quad v_{C r 2}=0 . \mathrm{O}$ transistor $T 1$ conduz e $i_{L r}, v_{C r 1}$, e $v_{C r 2}$ evoluem senoidalmente. Quando $v_{C r 2}=$ Vin o diodo $D 1$ é polarizado diretamente.

$2^{\underline{a}}$ Etapa ( $t 1, t 2 \rightarrow$ linear): $T 1, D 3, D 6, D 1$ estão em condução:

Condições iniciais: $i_{L r}=I 1 ; \quad v_{C r 2}=V i n ; \quad v_{C r 1}=0$. O diodo D1 entra em condução, a corrente $i_{L r}$ decresce linearmente até zero, as tensões $v_{C r l}$ e $v_{C r 2}$ se mantém nos mesmos valores.

$3^{\underline{a}}$ Etapa (t3, t4 $\rightarrow$ ressonante): T2, D4, D5 estão conduzindo:

Condições iniciais: $i_{L r}=0 ; \quad v_{C r l}=0 ; \quad v_{C r 2}=V i n$. Nesta etapa o mesmo modo de operação da $1^{\underline{a}}$ etapa é repetido.

$4^{\underline{a}}$ Etapa (t4, t5 $\rightarrow$ linear): T2, D4, D5, D2 estão em condução:

Condições iniciais: $i_{L r}=-I 1 ; \quad v_{C r 2}=V i n ; \quad v_{C r 1}=0$. A mesma operação apresentada na $2^{\underline{a}}$ etapa é aqui repetida.

Condições finais: $i_{L r}=0 ; \quad v_{C r 1}=V i n ; \quad v_{C r 2}=0$.

O bloqueio dos transistores $T 1$ e $T 2$ ocorre naturalmente, caracterizando uma comutação com corrente nula (ZCS). A figura 5 mostra as principais formas de onda relativas às quatro etapas de funcionamento.

\section{ANÁLISE QUANTITATIVA}

Através de uma análise matemática é possível determinar os esforços de tensão e/ou corrente sobre os componentes do circuito, assim como a sua característica de transferência de energia. Neste estudo serão apresentadas as curvas essenciais, de forma a tornar possível o estudo via simulação numérica.

\section{A. Obtenção do Plano de Fase}

Em regime permanente, apenas a análise das duas primeiras etapas (ressonante e linear) é suficiente para a construção do plano de fase desta estrutura.

$1^{\underline{a}}$ Etapa Ressonante (to, t1): A partir do circuito equivalente da figura 4.a e das condições iniciais iguais a zero, obtém-se a equação (3), que descreve o comportamento das grandezas na primeira etapa.

$2^{\underline{a}}$ Etapa Linear $(t 1, t 2)$ : A partir da figura $4 . \mathrm{b}$ e desprezando a queda de tensão nos diodos, determina-se a equação (5). Na figura 5, para $t=t 1 \Rightarrow I_{L r}(t)=I 1$, então (6) é obtida. Durante esta etapa a tensão $v_{C r l}$ é mantida no valor Vin.

$$
\begin{gathered}
V i n-E o p=V_{C r}(t)+L r \frac{d I_{L r}(t)}{d t} \\
V_{C r}(t)+j \sqrt{\frac{L r}{C r}} \cdot I_{L r}(t)=E-E \cdot e^{-j \omega_{o} t} \\
L r \cdot \frac{d I_{L r}(t)}{d t}+E o p=0 \\
I_{L r}(t)=I 1-\frac{E o p}{L r} \cdot t
\end{gathered}
$$

onde: $\omega_{O}=1 / \sqrt{L r \cdot C r} \quad ; \quad C r=C r 1+C r 2 \quad ; \quad Z o=\sqrt{L r / C r}$

$$
E=\text { Vin }- \text { Eop } \text {. }
$$

A partir das equações (3) e (4) pode-se construir o plano de fase da estrutura (figura 6).

\section{B. Curvas Relevantes e Expressões}

Devido à característica de fonte de corrente na saída, a potência deste conversor é dada pela soma das contribuições individuais de cada fase.

Considerando a potência de entrada igual à potência de saída, obtém-se:

$$
\text { Eop } \cdot\left(I o \cdot \frac{N s}{N p}\right)=3 \cdot P_{l \phi}
$$

onde $I o$ representa a corrente média de saída e $P_{1 \phi}$ a potência individual de cada fase (potência monofásica).

A corrente média de saída normalizada, referida ao primário do transformador, é definida pela equação (8).

$$
\overline{I o}=\frac{Z o}{\operatorname{Vin}} \cdot\left(\text { Io } \frac{N s}{N p}\right)
$$

Referindo-se a figura 6, é possível escrever:

$$
\begin{aligned}
& \cos \left(\pi-\alpha_{1}\right)=\frac{\operatorname{Vin}-E o p}{E}=\frac{E o p}{\operatorname{Vin}-E o p} \\
& \cos \left(\alpha_{1}\right)=\frac{-E o p}{\operatorname{Vin}-E o p}=\cos \left(\omega_{o} \cdot t 1\right)
\end{aligned}
$$

Durante a etapa ressonante a corrente de entrada é definida por:

$$
\operatorname{Iin}(t)=\frac{\operatorname{Vin}-E o p}{2 \cdot Z o} \cdot \operatorname{sen}\left(\omega_{o} t\right)
$$




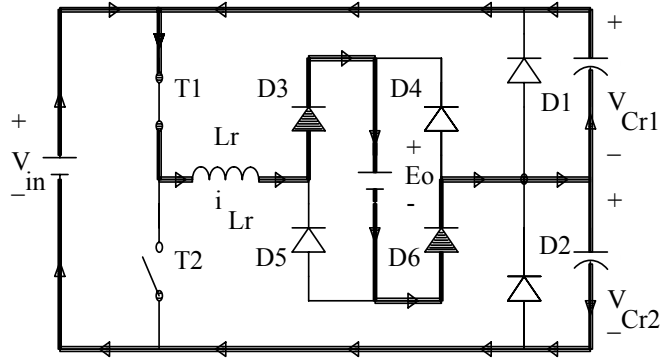

(a)

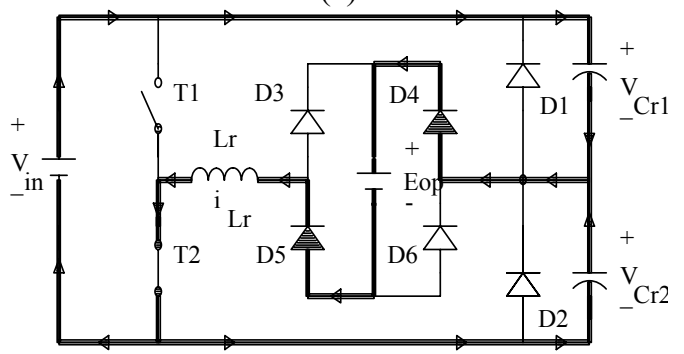

(c)

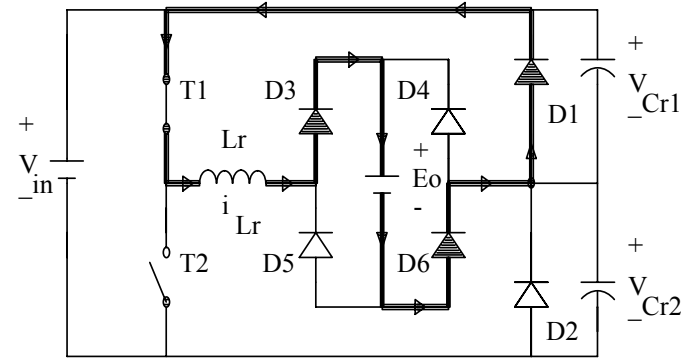

(b)

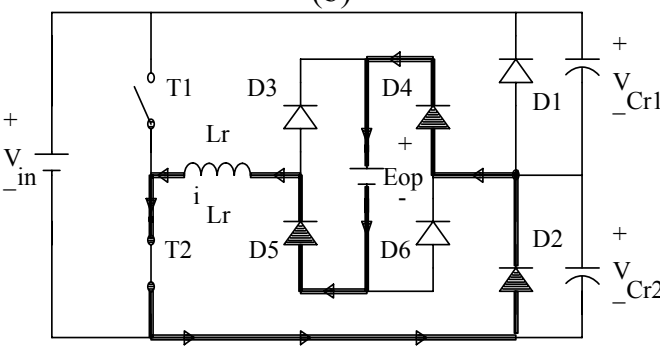

(d)

Figura 4: Etapas de Operação.

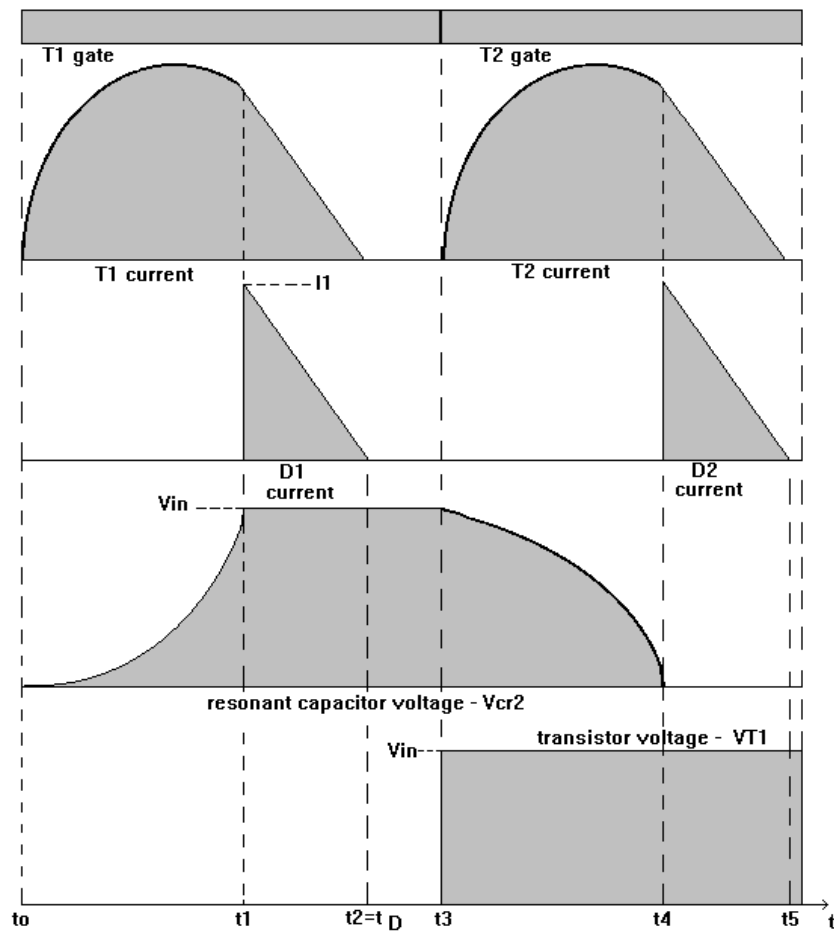

Figura 5: Principais Formas de Onda.

A corrente de entrada média instantânea é obtida por meio da seguinte expressão:

$$
\operatorname{Iin}_{\text {med }}=\frac{1}{T s} \int_{0}^{T s} \operatorname{Iin}(t) d t=\frac{2}{T s} \int_{0}^{t 1} \frac{\operatorname{Vin}-\text { Eop }}{2 \cdot \operatorname{Zo}} \cdot \operatorname{sen}\left(\omega_{o} t\right) d t
$$

onde: $\operatorname{Vin}=V m \cdot \operatorname{sen}(\omega t)$. Levando (10) em (12), obtém-se:

$$
\operatorname{Iin}_{\text {med }}=\frac{f_{s}}{2 \pi \cdot f o} \cdot \frac{V m}{Z o} \cdot \operatorname{sen} \theta_{1}
$$

Através das equações (7), (8), (13) e algumas manipulações, encontra-se a equação (14), que representa a corrente média de saída normalizada.

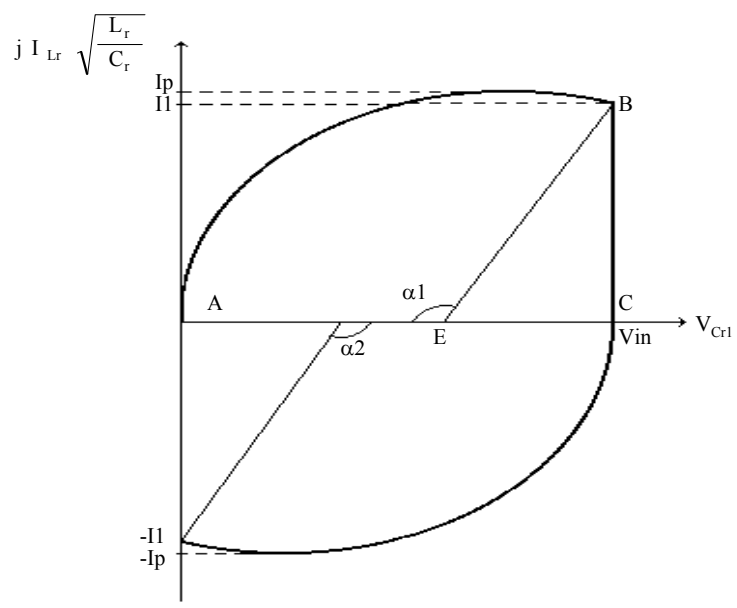

Figura 6: Plano de Fase.

$$
\overline{I o}=\frac{3 \cdot V m \cdot f s}{4 \pi^{2} \cdot f o \cdot E o p}\left(\pi-2 \operatorname{sen}^{-1} q+2 q \cdot \sqrt{1-q^{2}}\right)
$$

A equação (14) mostra que a corrente de saída é função da freqüência de operação e do ganho estático de tensão. Essa expressão prova que o controle do fluxo de energia é realizado através da modulação da freqüência. A figura 7.a mostra as curvas características do sistema, relacionando diferentes freqüências de chaveamento com o ganho estático. A linha tracejada representa o limite da condução continua. Os pontos de operação abaixo dessa linha indicam perda da comutação ZCS.

Substituindo a equação (2) em (15), a equação (16) é obtida. Essa expressão mostra que o fator de potência $(F P)$ da estrutura é unicamente função do ganho estático de tensão.

$$
F P=1-\frac{2 \theta_{1}-\operatorname{sen}\left(2 \theta_{1}\right)}{\pi}
$$

$$
F P=1-\frac{2 \operatorname{sen}^{-1} q-2 q \cdot \sqrt{1-q^{2}}}{\pi}
$$


Com a mesma relação entre freqüências, para um pequeno valor de $q$, obtém-se um excelente fator de potência para o conversor; mas isso implica em um acréscimo na corrente que circula pelos componentes do circuito, comprometendo o rendimento do sistema, devido ao aumento das perdas por condução. De acordo com as características desejadas, há uma relação ótima entre o $F P$ e $q$.

Especialmente nos casos de fontes de alimentação variáveis, a operação deve ser em condução descontínua, mesmo quando a tensão de entrada estiver no seu ponto de máximo (elevada energia armazenada no indutor ressonante). Logo, a relação entre a freqüência de operação e a freqüência de ressonância é determinada de forma a garantir que a comutação do transistor de potência se realize com corrente nula $($ Vin $=V m)$.

Durante o período de comutação há duas etapas ressonantes e duas etapas de roda-livre. A partir da figura 5 (desconsiderando o tempo morto e os pulsos de comando), a seguinte equação é obtida:

$$
T s_{\text {min }}=\frac{1}{f s_{\text {min }}}=2 t 1+2 t_{D}=2\left(t 1+t_{D}\right)
$$

onde: $T_{\text {Smim }} \rightarrow$ período mínimo para o modo de condução descontínua. Sendo que:

$$
t_{D}=t 2-t 1 \quad \text { e } \quad T o=\frac{1}{f_{o}}=\frac{2 \pi}{\omega_{o}}
$$

A equação (19) é obtida relacionando as freqüências com o ganho estático.

$$
\frac{f s_{\max }}{f_{0}}=\frac{\pi}{\cos ^{-1}\left(\frac{q}{q-2}\right)+\frac{2}{q} \sqrt{1-q}}
$$

$\mathrm{O}$ equacionamento para dimensionamento dos diodos e dos transistores é apresentado a seguir.

Corrente média normalizada nos diodos de grampeamento.

$$
\overline{I_{\text {GDmed }}}=\frac{f s}{4 \pi^{2} \cdot f o \cdot q} \cdot\left(\pi-2 \operatorname{sen}^{-1} q-2 q \cdot \sqrt{1-q^{2}}\right)
$$

onde: $I_{\text {GDmed }}=\overline{I_{\text {GDmed }}} \cdot \mathrm{Vm} / \mathrm{Zo}$, sendo $I_{\text {GDmed }}$ a corrente média nos diodos de grampeamento.

Corrente média normalizada nos interruptores de potência.

$$
\overline{I_{\text {Tmed }}}=\frac{f_{s}}{4 \pi^{2} \cdot f_{o} \cdot q} \cdot\left(\pi-2 \operatorname{sen}^{-1} q+2 q \sqrt{1-q^{2}}\right)
$$

Corrente eficaz normalizada nos diodos de grampeamento.

$$
{\overline{I_{G D e f}}}^{2}=\frac{4 f_{s}}{3 f_{0} \cdot \pi^{3} q} \cdot\left(1-q^{2}\right) \cdot(1-q)^{3 / 2}
$$

Corrente eficaz normalizada nos interruptores de potência.

$\bar{I}_{T e f}^{2}=\frac{f_{s}\left(1-q^{2}\right)}{2 \pi^{3} f o}\left[(2-q)^{2} \cos ^{-1} \frac{q}{q-2}+2 q \sqrt{1-q}+\frac{8}{3 q}(1-q)^{3 / 2}\right](23$

$$
\text { ) }
$$

Corrente de pico normalizada nos diodos de grampeamento.

$$
\overline{I_{G D \max }}=\sqrt{1-q}
$$

Corrente de pico normalizada nos interruptores de potência.

$$
\overline{I_{T \max }}=\frac{2-q}{2}
$$

Corrente eficaz no indutor ressonante.

$$
I_{L r}=\sqrt{I_{T 1 e f}^{2}+I_{T 2 e f}^{2}}=\sqrt{2} \cdot I_{T e f}
$$

A corrente nos diodos do retificador de saída é a mesma no interruptor de potência, multiplicada pela relação de transformação $(N p / N s)$. Portanto, as expressões (21), (22) e (23) são válidas para projeto dos retificadores de saída.

\section{Procedimento de Projeto e Exemplo}

De acordo com as características de entrada e saída desejadas, depois de escolher a tecnologia dos interruptores de potência, é possível projetar e simular o carregador de baterias. O projeto em malha aberta do conversor pode ser resumido em cinco passos (com $q$ fixado). Em nossa aplicação, o conversor é projetado para carregar quatro baterias conectadas em série, onde:

$$
\begin{array}{ll}
I o=55 \mathrm{~A} & \text { corrente média de saída; } \\
V e f=380 \mathrm{~V} & \text { tensão eficaz de entrada; } \\
f S_{\max }=30 \mathrm{kHz} & \text { freqüência máxima de chaveamento; } \\
F P_{\min }=0,95 & \text { fator de potência mínimo; } \\
E o=48 \mathrm{~V} & \text { tensão de saída. }
\end{array}
$$

$1^{\circ}$ Passo: A partir do $F P_{\min }$ e da figura 7.c ou equação (15), obtém-se o ângulo inicial $\theta_{l}$, que é o ângulo inicial para transferência de energia $\left(\theta_{1}=0,5 \mathrm{rad}=28,5^{\circ}\right)$.

$2^{\circ}$ Passo: Com o $F P_{\min }$ determina-se $q$ através de (16) $(q=$ $0,48)$.

$3^{\circ}$ Passo: Com $q$ é possível determinar a tensão primária do transformador e a relação de transformação $(N p / N s=$ Eop/Eo $),(E o p=128,4$ e $N p / N s=2,7)$.

$4^{\circ}$ Passo: Por meio da figura 7.b ou da equação (19), calculase a freqüência de ressonância $f o(f o \cong 47 \mathrm{kHz})$.

$5^{\circ}$ Passo: Com $f_{0}, f_{s}, F P_{\text {mim }}, q$, e as expressões (27) e (28) pode-se obter os parâmetros ressonantes:

$$
I o=\frac{3 f s \cdot F P_{\operatorname{mim}}}{2 \pi \cdot q \cdot f o \cdot \eta} \cdot \frac{V m}{\sqrt{L r / C r}} \cdot \frac{N p}{N s}
$$

$$
f o=\frac{1}{2 \pi \sqrt{L r \cdot C r}}
$$

Para um rendimento $\eta=0,95$, tem-se:

$$
Z o=16,75 \Omega ; L r=56,8 \mu \mathrm{H} ; C r=C r_{1}+C r_{2}=243 \mathrm{nF} \text {. }
$$

\section{RESULTADOS DE SIMULAÇÃO E EXPERIMENTAIS}

Os estudos via simulação tiveram como finalidade verificar a possibilidade de implementação do sistema proposto, principalmente no que se refere à ondulação de corrente na saída e à comutação $Z C S$ nos interruptores de potência.

A estrutura completa de potência, simulada e implementada, é apresentada na figura 8 e as especificações dos componentes são dadas abaixo, onde:
$L f_{1}=L f_{2}=L f_{3} \quad 470 \mu \mathrm{H}$
$L f_{11}=L f_{22}=L f_{33} \quad 135 \mu \mathrm{H}$;
$C f_{1}=C f_{2}=C f_{3} \quad 1,0 \mu \mathrm{F} / 250 \mathrm{~V} /$ polipropileno;
$C f_{1},=C f_{2},=C f_{3}, \quad 0,27 \mu \mathrm{F} / 400 \mathrm{~V} /$ polipropileno;
$L r_{1}=L r_{2}=L r_{3} \quad 46,4 \mu \mathrm{H}$ 
$\mathrm{Cr}_{1}=\mathrm{Cr}_{2}=\mathrm{Cr}_{3}$

$C r_{4}=C r_{5}=C r_{6}=119 \mu \mathrm{F} / 400 \mathrm{~V} /$ polipropileno;

Co

Lo

Eo

$R p$

$R c$

$1,2 \mu \mathrm{H}$;

$4 \times 12 \mathrm{~V}$ baterias;

$150 \mathrm{k} \Omega$;

$2,7 \Omega$;
DG1-DG6

DP1-DP6

$D 1-D 4=D 5-D 8=$

D9-D12

T1-T6
SKR e SKF 2F15/08, Semikron;

SKR 2F17/08, Semikron;
SE rificadora SKBB500C3200/ 2200, Semikron;

IGBT SKM40GB101D, Semikron.
$\overline{\mathrm{I}_{\mathrm{O}}}$

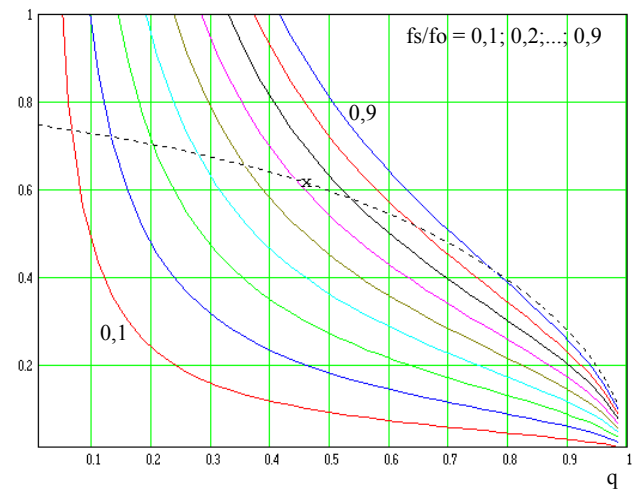

(a) Característica de saída

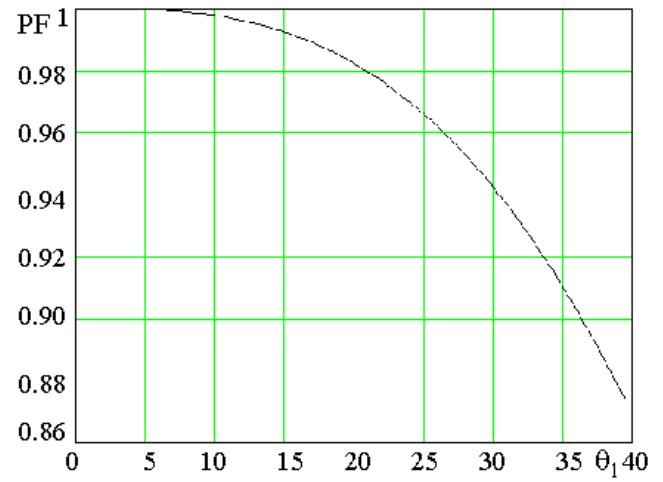

(c) Relação entre $F P$ e $\theta 1$.

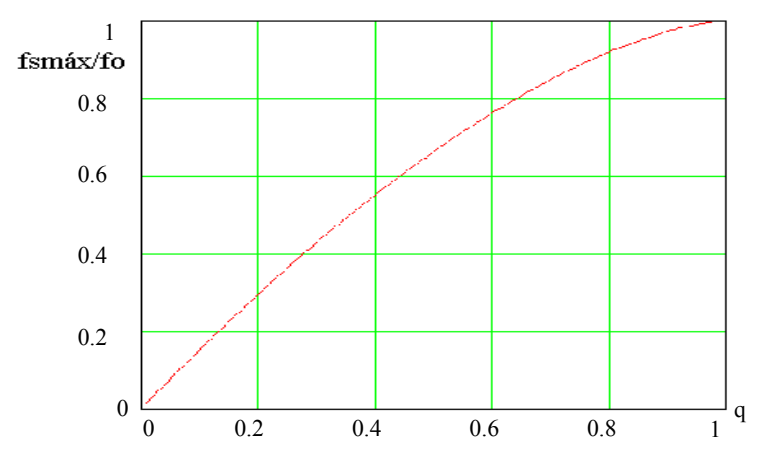

b) Relação $f s_{\max } / f o$ vs $q$

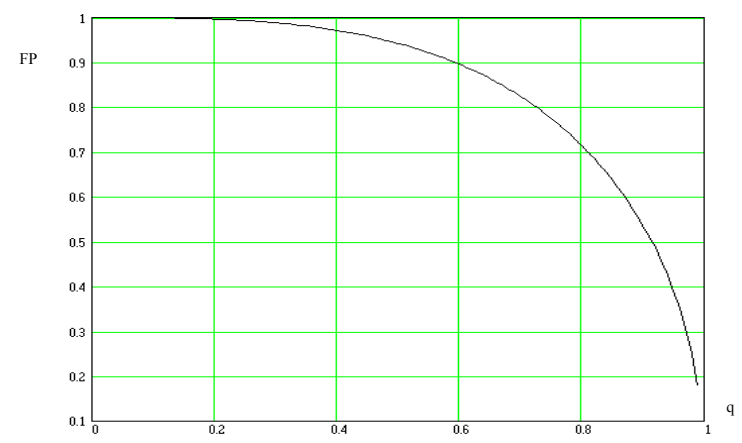

(d) Relação entre $F P$ e $q$.

Figura 7: Curvas para projeto.

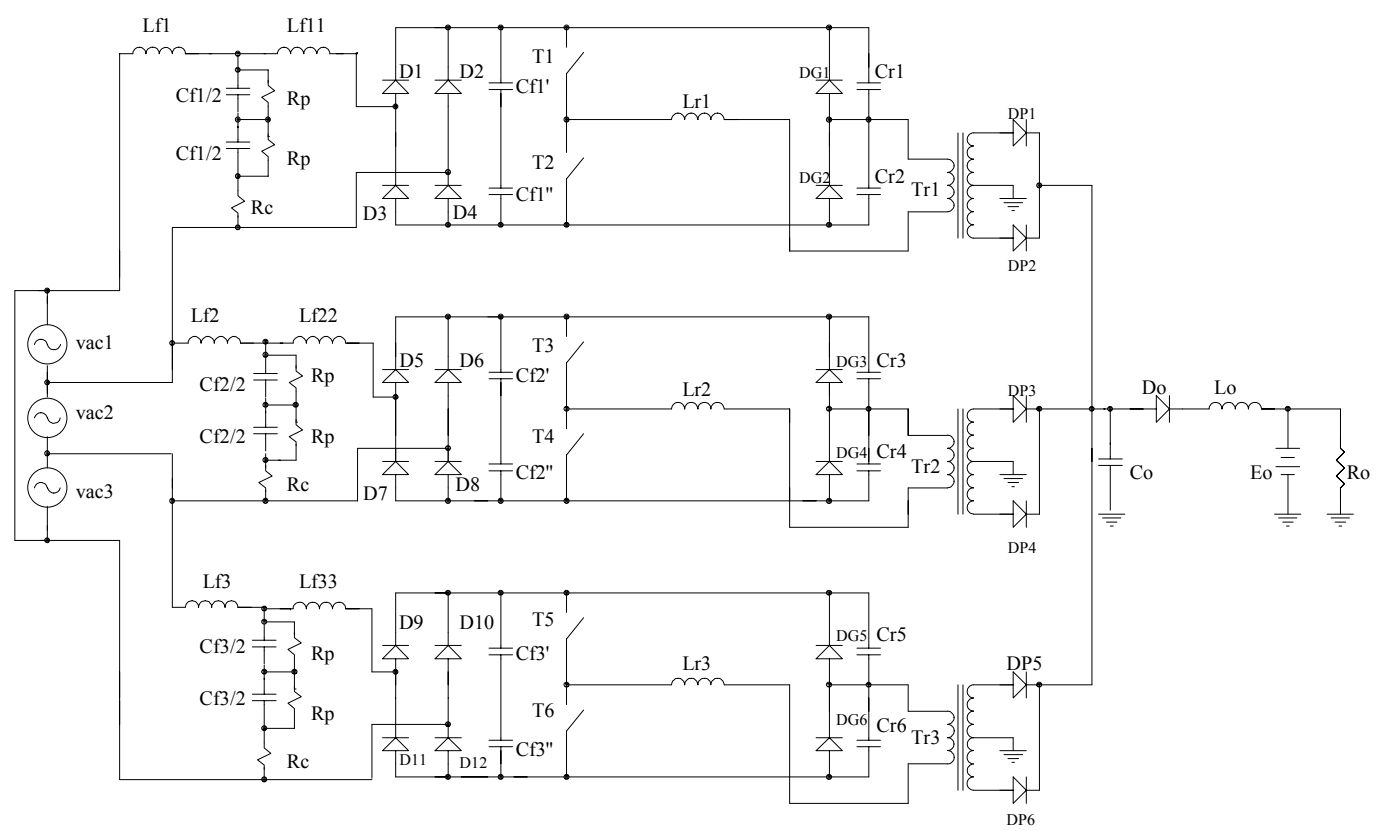

Figura 8: Estrutura de Potência implementada 


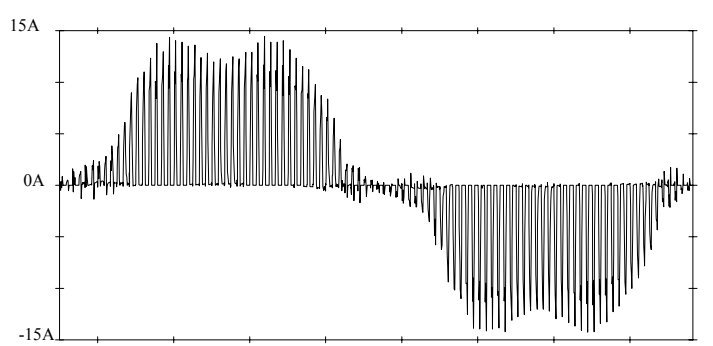

(5A/div, 2ms/div)

(a) Corrente de entrada.

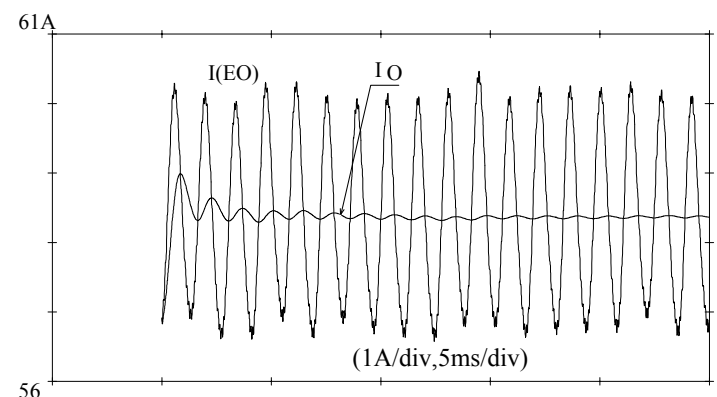

(c) Corrente de saída

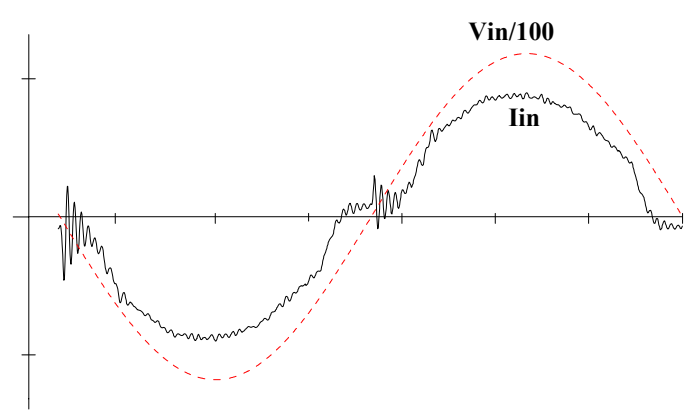

(5A/div;2,5ms/div)

(b) Tensão e corrente de entrada filtrada.

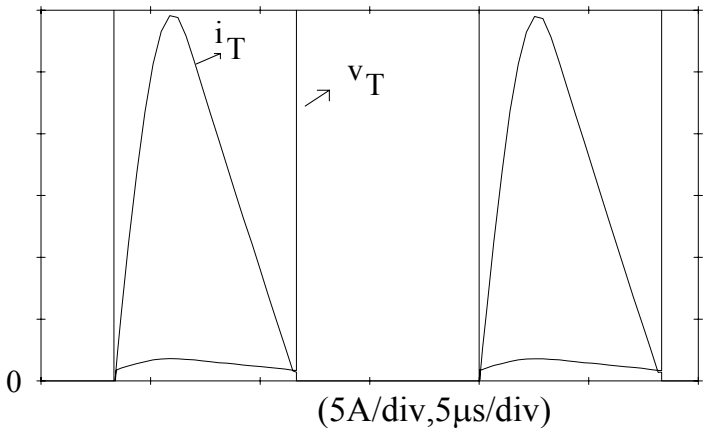

(d) Detalhe da comutação (ZCS).

Figura 9: (a,b,c,d) Resultados de simulação.

A figura 9 mostra as principais formas de onda obtidas por simulação usando o programa PROSCES [8], a qual apresenta como resultados: $P o \approx 2,8 \mathrm{~kW} ; F P \approx 0,98$ e $\theta_{l}=$ $0,52 \mathrm{rad}$.

As figuras 9.a e 9.b mostram a corrente de entrada na fase 1 , sem e com filtro, respectivamente. A freqüência dessa corrente é de $60 \mathrm{~Hz}$, mas é modulada com o dobro da freqüência de chaveamento.

A figura 9.c apresenta o valor médio e a ondulação de corrente na saída do conversor, cujos valores são respectivamente $58 \mathrm{~A}$ e $3 \mathrm{~A}$.

O detalhe da comutação está representado na figura 9.d. Verifica-se que os interruptores principais comutam em $Z C S$.

$\mathrm{Na}$ figura 10.a tem-se a tensão de linha e a corrente filtrada de linha. É possível observar que a tensão da rede disponível no laboratório tem uma distorção harmônica e a corrente segue essa distorção. A Tabela I apresenta os valores medidos a partir de um protótipo de laboratório. Os resultados obtidos são coerentes com os da simulação.

A figura 10.b mostra a tensão e a corrente no circuito ressonante. Verifica-se que a corrente magnetizante é muito pequena, os valores medidos estão em torno de $600 \mathrm{~mA}$.

A corrente de saída é apresentada na figura 10.c. Uma ondulação em $360 \mathrm{~Hz}(\Delta \mathrm{I} \approx 2,5 \mathrm{~A})$ é observada devido principalmente ao fato que a corrente de saída é a soma de três correntes defasadas de $120^{\circ}$.

A característica de comutação ZVS fica comprovada na figura 10.d, onde são apresentadas a tensão e a corrente no IGBT para a máxima potência de saída.

A curva de rendimento $(\eta \times f s)$ para $q$ fixo, é apresentada na figura 11. As perdas mais relevantes ocorreram no retificador de saída e nos elementos magnéticos.

\section{CONCLUSÕES}

O conversor estudado neste artigo comporta-se de acordo com a análise matemática previamente desenvolvida. Ele apresenta alto fator de potência sem a necessidade de circuitos intermediários, o que o torna um sistema muito simples de ser implementado. O circuito de comando gera pulsos complementares que acionam cada um dos interruptores de cada fase instantaneamente, mantendo a isolação.

Os conversores em cada fase são independentes, facilitando o projeto do sistema e aumentando a confiabilidade global, tendo em vista que a perda de um dos módulos de uma fase, mantém o sistema operando, embora com $30 \%$ a menos de potência. Além disso, devido a configuração utilizada é possível o uso de módulos padronizados. A freqüência de operação determina a potência de saída.

Como características negativas tem-se: fluxo de potência pulsante e elevado número de componentes.

A característica de fonte de corrente na saída oferece facilmente a possibilidade de associação em paralelo. A entrada do tipo trifásica permite diminuir drasticamente a ondulação da corrente de saída

Finalmente os autores consideram a estrutura proposta particularmente interessante para aplicações industriais. 


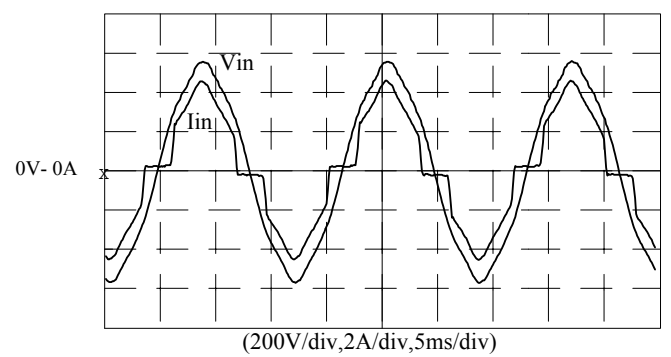

(a) Tensão de entrada e corrente de entrada filtrada.

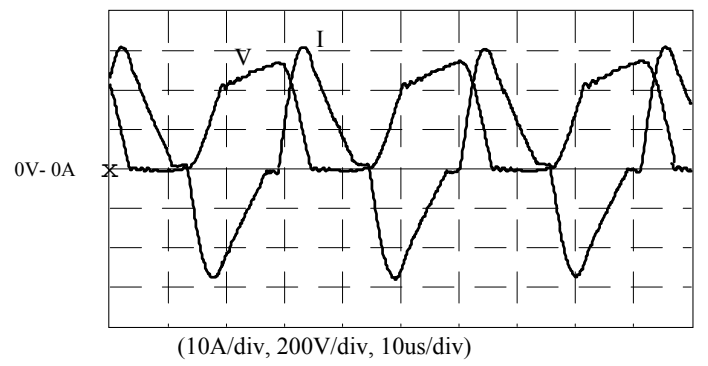

(b) Tensão e corrente no circuito ressonante.

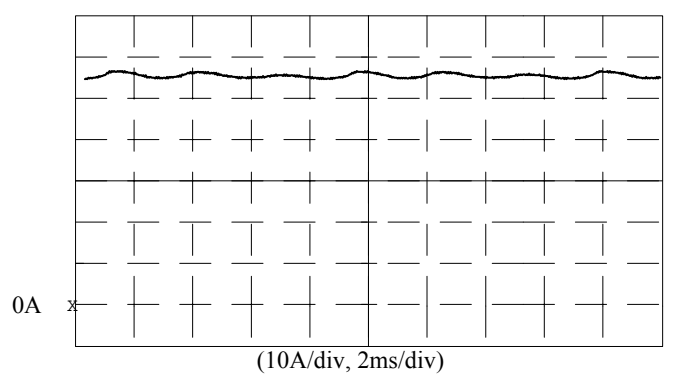

(c) Corrente de saída.

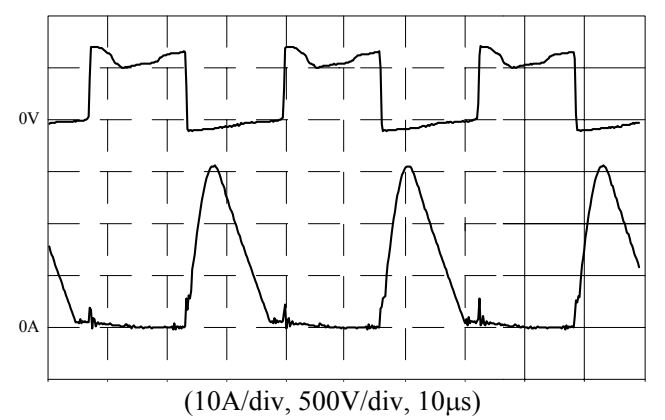

(d) Tensão e corrente no IGBT.

Figura 10. Resultados Experimentais.

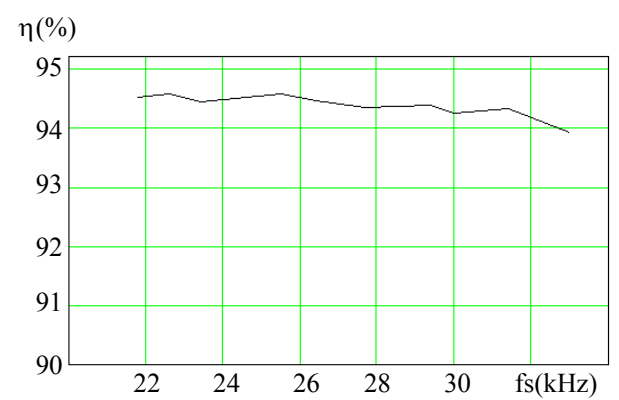

Fig. 11 - Curva de rendimento.
TABELA I: Valores medidos para condições nominais.

\begin{tabular}{|c|c|c|c|c|}
\hline fase & $\mathrm{I}_{\mathrm{RMS}}$ & DHT I(\%) & DHT V(\%) & FP \\
\hline $\mathrm{R}$ & 4.01 & 23,6 & 3,4 & 0,973 \\
\hline $\mathrm{S}$ & 4.02 & 22,35 & 3,4 & 0,976 \\
\hline $\mathrm{T}$ & 4.06 & 20,76 & 3,3 & 0,980 \\
\hline
\end{tabular}

\section{REFERÊNCIAS BIBLIOGRÁFICAS}

[1] D. Gauger et al., "A Three-phase off-line Switching Power Supply With Unity Power Factor and Low TIF", IEEE INTELEC'86 Conf. Rec., pp. 115-121, Oct. 1986.

[2] T. Latos, D. Basack, "A High Efficiency $3 \mathrm{~kW}$ Switching Battery Charger”, IEEE PESC'82 Conf. Rec., pp. 341349, June 1982.

[3] B. Ignazia, "Unity Power Factor Battery Charger by LVI", in Power Quality Proc., pp. 42-47, Nov. 1990.

[4] D. Simonetti, J. Sebastian, J. Uceda, "Single-Switch Three Phase Power Pre-Regulator Under Variable Switching Frequency and Discontinuous Input Current", IEEE PESC'93 Conf. Rec., pp. 657-661, June 1993.

[5] P. Ziogas, S. Manias, A. Prasad, "An Active Power Factor Correction Technique For Three Phase Diode Rectifiers", IEEE PESC'89 Conf. Rec., pp. 58-65, June 1989.

[6] E. Ismail, R. W. Erickson, "A Single Transistor Three Phase Resonant Switch For High Quality Rectification", IEEE PESC'92 Conf. Rec., pp. 1341-1351, June 1992.

[7] J. Pforr, L. Hobson, "A Novel Power Factor Corrected Single Ended Resonant Converter With Three Phase Supply", IEEE PESC'92 Conf. Rec., pp. 1369-1375, June 1992.

[8] D. C. Martins, et al., "Performance Study of PROSCES/MACHINE”, IEE-PEVD'96 Conf. Rec., pp. 502-507, September 1996.

\section{DADOS BIOGRÁFICOS}

Denizar Cruz Martins, nasceu em São Paulo, SP, em 24 de Abril de 1955. Formou-se em Engenharia Elétrica e obteve o título de Mestre em Engenharia Elétrica pela Universidade Federal de Santa Catarina, Florianópolis - SC em 1978 e 1981, respectivamente. Concluiu o Doutorado no INPT, Toulouse - França, em 1986. Atualmente é professor titular do Depto. de Engenharia Elétrica da Universidade Federal de Santa Catarina, Florianópolis - SC. O Prof. Denizar já publicou mais de 100 trabalhos científicos entre revistas e congressos nacionais e internacionais, realizou mais de 30 consultorias técnicas e obteve 02 patentes de invenção e um registro de software. Sua área de atuação compreende: desenvolvimento de conversores para tratamento de energia solar com alta qualidade de energia, conversores de alta freqüência e simulação de conversor estáticos. É membro da SOBRAEP, da SBA e do IEEE.

Elias Sebastião de Andrade, nasceu em Florianópolis, SC, em 23 de agosto de 1965. Formou-se em Engenharia Elétrica e obteve o título de Mestre em Engenharia Elétrica pela Universidade Federal de Santa Catarina, Florianópolis - SC em 1992 e 1994, respectivamente. Sua área de interesse concentra-se em conversores de alta freqüência e alto fator de potência. 